\title{
Zero-temperature phases for chiral magnets in three dimensions
}

\author{
Jin-Hong Park ${ }^{1}$ and Jung Hoon Han $^{1}$ \\ ${ }^{1}$ Department of Physics and BK21 Physics Research Division, \\ Sungkyunkwan University, Suwon 440-746, Korea
}

(Dated: September 10, 2018)

\begin{abstract}
We calculate the energies of various multiple-spiral spin configurations for the three-dimensional model of chiral magnets. The ground-state phase diagram is obtained in the space of anisotropy, magnetic field, and interaction parameters. Regimes with multiple-spiral, or spin crystal ground states of two- and three-dimensional nature are identified. The Skyrmion number responsible for anomalous Hall effect is examined for each spin crystal configuration. A geometric interpretation is given for the three-dimensional spin crystal lattice structures as the periodic arrangement of hedgehog and anti-hedgehog singularities.
\end{abstract}

PACS numbers: $75.10 . \mathrm{Hk}, 75.25 . \mathrm{Aj}, 75.30 . \mathrm{Kz}, 75.50 . \mathrm{Bb}$

\section{INTRODUCTION}

Skyrmionic spin texture is a natural excitation of a ferromagnet in two spatial dimensions (2D) carrying nontrivial topological numbers ${ }^{1,2}$. Although their creation energy remains finite (of order of several $J$, $J=$ ferromagnetic exchange energy) and can be excited thermally, an important work of Polaykov ${ }^{3,4}$ demonstrated that non-topological spin-wave excitations already destroy order at all finite temperatures, preemptying the chance of a phase transition driven by topological defects. This is in contrast to the XY spin model in the same dimension, where the transition to disordered phase indeed occurs by proliferation of topological defects known as vortices ${ }^{5}$.

Recent advances in the experimental investigation of chiral magnets ${ }^{6-11}$ brought new insights into the role and importance of Skyrmions in the class of magnets characterized by both Heisenberg $(J)$ and DzyaloshinskiiMoriya $(D)$ interactions. A most interesting finding of the recent experiments is that a rather small magnetic field of order $D^{2} / J$ leads to the proliferation of Skyrmions and their crystallization into a columnar $(3 \mathrm{D})^{6,12,13}$ or a pancake-like $(2 \mathrm{D})^{9}$ triangular array, depending on the dimensionality. The stability of the triangular Skyrmion lattice was successfully demonstrated based on Ginzburg-Landau theory ${ }^{6}$ and on microscopic model calculations ${ }^{14,15}$.

Much less understood aspect of Skyrmion physics in chiral magnets is whether it is possible to realize other forms of Skyrme crystalline order. Indeed it was argued that a square array of Skyrmions and anti-Skyrmions would be possible energetically ${ }^{14,16,17}$. In three dimensions, study of different 3D crystalline structures was pioneered by Binz and collaborators ${ }^{18}$. In this paper, we carry out a comprehensive study of the energetic stability of many different kinds of spin crystal configurations in three spatial dimensions, under the influence of a number of spin anisotropy interactions and magnetic field. Our calculation focuses on zero-temperature energetics and differs from other considerations where temperature control also played a vital part ${ }^{6,16,17}$. It also differs from Binz et al. ${ }^{18}$ in that the role of magnetic field in stabilizing Skyrmion order is more thoroughly examined here.

Some conclusions reached in our paper are as follows. (i) Two-dimensional multiple-spiral structures are energetically unfavorable in three spatial dimensions. (ii) For strong interactions fully three-dimensional spin crystal phase becomes more stable compared to single-spiral phases. Typically, magnetic field tends to stabilize 3D spin crystal structures. (iii) All 3D multiple-spiral structures can be understood as a lattice of hedgehogs and anti-hedgehogs. Such identification allows a complicated, but still straightforward evaluation of the Skyrmion number. Organization of the paper begins by introducing several multiple spiral states in Sec. II along with some single spiral states. The free energy for each state is worked out following the detailed procedure described in the Appendix. In Sec. III the ground state phase diagram is worked out in search for the energetic stability of multiple spiral phases in three dimensions. We also carry out a calculation of the spin chirality (Skyrmion number) for each of the multiple spiral phases in Sec. IV. A useful visual interpretation is given of the three-dimensional Skyrmion crystals as a lattice of hedgehog singularities. Finally we conclude with a summary in Sec. V.

Throughout the paper, the expression "multiple spiral" state is used interchangeably with "spin crystal" state. A single spiral state does not form a spin crystal.

\section{MULTIPLE SPIRAL STATE MODEL}

The multiple spiral spin configuration can be written in the general form

$$
\begin{aligned}
\mathbf{n}_{\mathbf{r}} & =\mathbf{n}_{\mathbf{0}}+\mathbf{\Phi}_{\mathbf{r}} \\
\boldsymbol{\Phi}_{\mathbf{r}} & =\sum_{\alpha=1}^{N}\left(\mathbf{n}_{\alpha} e^{i \mathbf{k}_{\alpha} \cdot \mathbf{r}}+\mathbf{n}_{\alpha}^{*} e^{-i \mathbf{k}_{\alpha} \cdot \mathbf{r}}\right),
\end{aligned}
$$

as the sum of a uniform magnetization $\mathbf{n}_{0}=\left(0,0, n_{0}\right)$ due to the Zeeman field and $N$ independent spirals of wave 
vectors $\mathbf{k}_{\alpha}, \alpha=1 \cdots N$. It is assumed that all $\mathbf{k}_{\alpha}$ 's are of the same length, and the amplitudes $\mathbf{n}_{\alpha} \cdot \mathbf{n}_{\alpha}^{*}=n_{H}^{2} / 2$ are the same for all the spiral modes. The magnitude of the spin is position-dependent, and we impose the average normalization on $\mathbf{n}_{\mathbf{r}}$

$$
\left\langle\mathbf{n}_{\mathbf{r}}^{2}\right\rangle=1=\mathbf{n}_{0}^{2}+2 \sum_{\alpha} \mathbf{n}_{\alpha} \cdot \mathbf{n}_{\alpha}^{*}=n_{0}^{2}+N n_{H}^{2} .
$$

In this sense, our spins are "soft", with the origin of softness possibly traced to the coupling of spins to the conduction electron bath or the averaging over fast fluctuations.

For right-handed spirals we can choose

$$
\mathbf{n}_{\alpha}=\frac{n_{H}}{2} e^{i \theta_{\alpha}}\left(\hat{e}_{1 \alpha}-i \hat{e}_{2 \alpha}\right)
$$

using a pair of orthonormal vectors forming a triad, $\hat{e}_{1 \alpha} \times$ $\hat{e}_{2 \alpha}=\hat{k}_{\alpha}$. An arbitrary phase $\theta_{\alpha}$ is assigned for each spiral mode for generality.

Our consideration of the energy of multiple spiral spins is based on the free energy

$$
\begin{aligned}
F[\mathbf{n}] & =\int d^{3} \mathbf{r} f[\mathbf{n}], \\
f[\mathbf{n}] & =\frac{1}{2} A\left(n_{x}^{2}+n_{y}^{2}+n_{z}^{2}\right)+D \mathbf{n} \cdot(\boldsymbol{\nabla} \times \mathbf{n})-\mathbf{B} \cdot \mathbf{n} \\
& +\frac{1}{2} J\left[\left(\nabla n_{x}\right)^{2}+\left(\nabla n_{y}\right)^{2}+\left(\nabla n_{z}\right)^{2}\right] \\
& +\frac{1}{2} C\left[\left(\frac{\partial n_{x}}{\partial x}\right)^{2}+\left(\frac{\partial n_{y}}{\partial y}\right)^{2}+\left(\frac{\partial n_{z}}{\partial z}\right)^{2}\right] \\
& +U\left(n_{x}^{2}+n_{y}^{2}+n_{z}^{2}\right)^{2}+W\left(n_{x}^{4}+n_{y}^{4}+n_{z}^{4}\right) .
\end{aligned}
$$

Ferromagnetic exchange and Dzyaloshinskii-Moriya (DM) interactions are given by $J$ and $D$ while $C, U$, and $W$ are the spin anisotropy and interaction terms up to fourth order introduced by Bak and Jensen ${ }^{19}$. Zeeman field $\mathbf{B}$ is always directed along the $\hat{z}$-direction in this paper. The $A$-term can be dropped in practice because we will always require average magnetization of unity, which also makes our calculation effectively zerotemperature. The integration $\int d^{3} \mathbf{r}$ extends over threedimensional space and is divided by the space volume.

If we remove all but $J, D$, and $\mathbf{B}$ from the free energy, the multiple spiral state defined in Eq. (1) can be readily shown to have the energy

$$
E=\frac{1}{2} N n_{H}^{2}\left(J k^{2}-2 D k\right)-B n_{0}
$$

Upon optimizing $k$ at $k=D / J$ it becomes

$$
E=-\frac{1}{2} \frac{D^{2}}{J} N n_{H}^{2}-B n_{0}=\frac{1}{2} \frac{D^{2}}{J}\left(n_{0}^{2}-1\right)-B n_{0}
$$

Minimizing it further in terms of $n_{0}$ gives

$$
n_{0}=\frac{B}{D^{2} / J}, \quad E=-\frac{1}{2} \frac{B^{2}}{D^{2} / J} .
$$

It is interesting that all multiple spiral states of the type (1), regardless of the number of spiral components $N$ and their orientations, carry exactly the same $\mathbf{k}$-vector length, magnetization $n_{0}$, and the ground state energy, in the presence of magnetic field.

It is then up to the anisotropy and interaction terms $C, U, W$ to separate out the energies of different multiple spiral states. Below we work with eight exemplary spin configurations, drawn from both recent experiments and other theories, with $N$, the number of spiral components, ranging from 1 to 6 . For $N>1$ one will obtain spin crystal state of some sort.

First, three kinds of $N=1$ single spiral states are considered.

- Helical spin state with $\mathbf{k}=(k / \sqrt{3})(1,1,1)$ has the spin configuration

$$
\begin{aligned}
& \mathbf{n}_{\mathbf{r}}^{\mathrm{H}_{111}}=\mathbf{n}_{0}+\mathbf{n}_{1}^{\mathrm{H}_{111}} e^{i \mathbf{k} \cdot \mathbf{r}}+\left(\mathbf{n}_{1}^{\mathrm{H}_{111}}\right)^{*} e^{-i \mathbf{k} \cdot \mathbf{r}}, \\
& \mathbf{n}_{1}^{\mathrm{H}_{111}}=\frac{n_{H}}{2} e^{i \theta}\left(\frac{\hat{x}+\hat{y}-2 \hat{z}}{\sqrt{6}}+i \frac{\hat{x}-\hat{y}}{\sqrt{2}}\right) .
\end{aligned}
$$

This state appears as the ground state at low magnetic field $\left(B<B_{c}\right)$ for bulk MnSi crystal ${ }^{6-8}$.

- Another helical state has $\mathbf{k}=(k / \sqrt{2})(1,1,0)$ and the spin configuration

$$
\begin{aligned}
\mathbf{n}_{\mathbf{r}}^{\mathrm{H}_{110}} & =\mathbf{n}_{0}+\mathbf{n}_{1}^{\mathrm{H}_{110}} e^{i \mathbf{k} \cdot \mathbf{r}}+\left(\mathbf{n}_{1}^{\mathrm{H}_{110}}\right)^{*} e^{-i \mathbf{k} \cdot \mathbf{r}}, \\
\mathbf{n}_{1}^{\mathrm{H}_{110}} & =\frac{n_{H}}{2} e^{i \theta}\left(\hat{z}+i \frac{\hat{y}-\hat{x}}{\sqrt{2}}\right) .
\end{aligned}
$$

This state appears as the low-field ground state in the $2 \mathrm{D}$ geometry ${ }^{9,14,15}$.

- Conical state has $\mathbf{k}=k(0,0,1)$ and the spin configuration

$$
\begin{aligned}
& \mathbf{n}_{\mathbf{r}}^{\mathrm{Co}}=\mathbf{n}_{0}+\mathbf{n}_{1}^{\mathrm{Co}} e^{i \mathbf{k} \cdot \mathbf{r}}+\left(\mathbf{n}_{1}^{\mathrm{Co}}\right)^{*} e^{-i \mathbf{k} \cdot \mathbf{r}} \\
& \mathbf{n}_{1}^{\mathrm{Co}}=\frac{n_{H}}{2} e^{i \theta}(\hat{x}-i \hat{y})
\end{aligned}
$$

It includes the ferromagnetic (FM) state as a special case. This is the ground state for $3 \mathrm{D} \mathrm{MnSi}$ at fields $B>B_{c}{ }^{6-8}$.

With $N=2$ and $N=3$ we consider two kinds of twodimensional Skyrme crystal states extended along the $z$ direction in a columnar manner. 
- Square-lattice Skyrmion crystal $(\operatorname{SkX} 2)(N=2)$ has the propagation vectors $\mathbf{k}_{1}=k(1,0,0), \mathbf{k}_{2}=$ $k(0,1,0)$, and the spin configuration

$$
\begin{aligned}
& \mathbf{n}_{\mathbf{r}}^{\mathrm{SkX} 2}=\mathbf{n}_{0}+\sum_{\alpha}\left(\mathbf{n}_{\alpha}^{\mathrm{SkX} 2} e^{i \mathbf{k}_{\alpha} \cdot \mathbf{r}}+\left(\mathbf{n}_{\alpha}^{\mathrm{SkX} 2}\right)^{*} e^{-i \mathbf{k}_{\alpha} \cdot \mathbf{r}}\right), \\
& \mathbf{n}_{1}^{\mathrm{SkX} 2}=\frac{n_{H}}{2} e^{i \theta_{1}}(\hat{y}-i \hat{z}), \\
& \mathbf{n}_{2}^{\mathrm{SkX} 2}=\frac{n_{H}}{2} e^{i \theta_{2}}(\hat{z}-i \hat{x}) .
\end{aligned}
$$

The spin configuration is that of half-Skyrmions and half-anti-Skyrmions alternating on a square lattice. Such a state was previously considered in Refs. 14,16,17. Its existence in nature remains elusive so far.

- Triangular-lattice SkX state $(N=3)$ has $\mathbf{k}_{1}=k(1,0,0), \mathbf{k}_{2}=k(-1 / 2, \sqrt{3} / 2,0), \mathbf{k}_{3}=$ $k(-1 / 2,-\sqrt{3} / 2,0)$, and the spin configuration

$$
\begin{aligned}
& \mathbf{n}_{\mathbf{r}}^{\mathrm{SkX} 3}=\mathbf{n}_{0}+\sum_{\alpha}\left(\mathbf{n}_{\alpha}^{\mathrm{SkX} 3} e^{i \mathbf{k}_{\alpha} \cdot \mathbf{r}}+\left(\mathbf{n}_{\alpha}^{\mathrm{SkX} 3}\right)^{*} e^{-i \mathbf{k}_{\alpha} \cdot \mathbf{r}}\right), \\
& \mathbf{n}_{1}^{\mathrm{SkX} 3}=\frac{n_{H}}{2} e^{i \theta_{1}}(\hat{z}+i \hat{y}), \\
& \mathbf{n}_{2}^{\mathrm{SkX} 3}=\frac{n_{H}}{2} e^{i \theta_{2}}\left(\hat{z}-i \frac{\sqrt{3}}{2} \hat{x}-i \frac{\hat{y}}{2}\right), \\
& \mathbf{n}_{3}^{\mathrm{SkX} 3}=\frac{n_{H}}{2} e^{i \theta_{3}}\left(\hat{z}+i \frac{\sqrt{3}}{2} \hat{x}-i \frac{\hat{y}}{2}\right) .
\end{aligned}
$$

This state was found to exist in the A-phase of $\mathrm{MnSi}$ and related B20-compounds ${ }^{6-8}$ as well as in the $2 \mathrm{D}$ thin-film sample at low temperature in the presence of magnetic field ${ }^{9}$.

Another possible spin crystal arrangement with $N=3$ is the simple-cubic (SC) multiple-spiral spin structure ${ }^{18}$ which consists of $\mathbf{k}_{1}=k(1,0,0), \mathbf{k}_{2}=k(0,1,0)$ and $\mathbf{k}_{3}=$ $k(0,0,1)$.

$$
\begin{aligned}
& \mathbf{n}_{\mathbf{r}}^{\mathrm{SC}}=\mathbf{n}_{0}+\sum_{\alpha}\left(\mathbf{n}_{\alpha}^{\mathrm{SC}} e^{i \mathbf{k}_{\alpha} \cdot \mathbf{r}}+\left(\mathbf{n}_{\alpha}^{\mathrm{SC}}\right)^{*} e^{-i \mathbf{k}_{\alpha} \cdot \mathbf{r}}\right), \\
& \mathbf{n}_{1}^{\mathrm{SC}}=\frac{n_{H}}{2} e^{i \theta_{1}}(\hat{y}-i \hat{z}), \\
& \mathbf{n}_{2}^{\mathrm{SC}}=\frac{n_{H}}{2} e^{i \theta_{2}}(\hat{z}-i \hat{x}), \\
& \mathbf{n}_{3}^{\mathrm{SC}}=\frac{n_{H}}{2} e^{i \theta_{3}}(\hat{x}-i \hat{y}) .
\end{aligned}
$$

At $N=4$ one obtains face-centered-cubic (FCC) multiple spiral spin structure ${ }^{18}$ consisting of $\mathbf{k}_{1}=$ $(k / \sqrt{3})(1,1,1), \quad \mathbf{k}_{2}=(k / \sqrt{3})(-1,-1,1), \quad \mathbf{k}_{3}=$ $(k / \sqrt{3})(-1,1,-1), \mathbf{k}_{4}=(k / \sqrt{3})(1,-1,-1)$, and the spin configuration
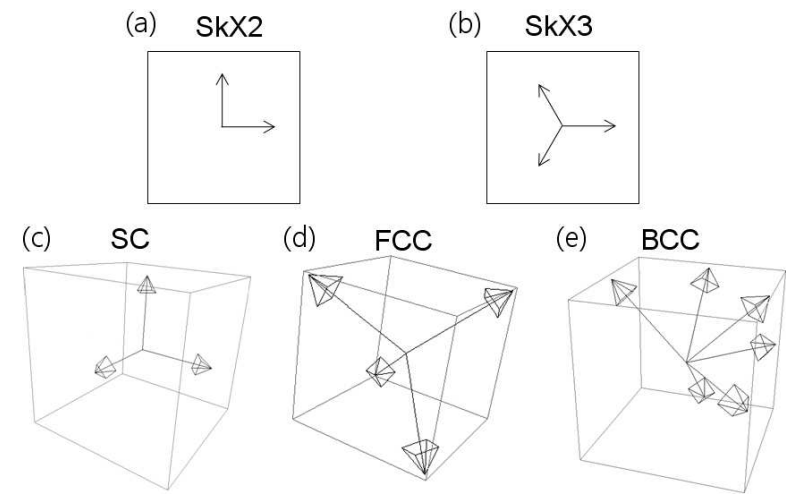

FIG. 1: The k-vectors of five multiple-spiral spin states considered in this paper. (a) and (b) are the k-vectors of SkX2 and SkX3 in two dimensions. (c), (d) and (e) are the $\mathbf{k}$-vectors of SC, FCC and BCC spin crystal states, respectively.

$$
\begin{aligned}
& \mathbf{n}_{\mathbf{r}}^{\mathrm{FCC}}=\mathbf{n}_{0}+\sum_{\alpha}\left(\mathbf{n}_{\alpha}^{\mathrm{FCC}} e^{i \mathbf{k}_{\alpha} \cdot \mathbf{r}}+\left(\mathbf{n}_{\alpha}^{\mathrm{FCC}}\right)^{*} e^{-i \mathbf{k}_{\alpha} \cdot \mathbf{r}}\right), \\
& \mathbf{n}_{1}^{\mathrm{FCC}}=\frac{n_{H}}{2} e^{i \theta_{1}}\left(\frac{\hat{x}+\hat{y}-2 \hat{z}}{\sqrt{6}}+i \frac{\hat{x}-\hat{y}}{\sqrt{2}}\right), \\
& \mathbf{n}_{2}^{\mathrm{FCC}}=\frac{n_{H}}{2} e^{i \theta_{2}}\left(\frac{\hat{x}+\hat{y}+2 \hat{z}}{\sqrt{6}}+i \frac{\hat{x}-\hat{y}}{\sqrt{2}}\right), \\
& \mathbf{n}_{3}^{\mathrm{FCC}}=\frac{n_{H}}{2} e^{i \theta_{3}}\left(\frac{\hat{x}+2 \hat{y}+\hat{z}}{\sqrt{6}}+i \frac{\hat{z}-\hat{x}}{\sqrt{2}}\right), \\
& \mathbf{n}_{4}^{\mathrm{FCC}}=\frac{n_{H}}{2} e^{i \theta_{4}}\left(\frac{2 \hat{x}+\hat{y}+\hat{z}}{\sqrt{6}}+i \frac{\hat{y}-\hat{z}}{\sqrt{2}}\right) .
\end{aligned}
$$

Finally, at $N=6$ one obtains body-centeredcubic (BCC) multiple spiral states ${ }^{18}$ consisting of $\mathbf{k}_{1}=(k / \sqrt{2})(1,1,0), \quad \mathbf{k}_{2}=(k / \sqrt{2})(1,-1,0)$, $\mathbf{k}_{3}=(k / \sqrt{2})(1,0,1), \mathbf{k}_{4}=(k / \sqrt{2})(-1,0,1), \mathbf{k}_{5}=$ $(k / \sqrt{2})(0,1,1), \mathbf{k}_{6}=(k / \sqrt{2})(0,1,-1)$, and the spin configuration

$$
\begin{aligned}
& \mathbf{n}_{\mathbf{r}}^{\mathrm{BCC}}=\mathbf{n}_{0}+\sum_{\alpha}\left(\mathbf{n}_{\alpha}^{\mathrm{BCC}} e^{i \mathbf{k}_{\alpha} \cdot \mathbf{r}}+\left(\mathbf{n}_{\alpha}^{\mathrm{BCC}}\right)^{*} e^{-i \mathbf{k}_{\alpha} \cdot \mathbf{r}}\right), \\
& \mathbf{n}_{1}^{\mathrm{BCC}}=\frac{n_{H}}{2} e^{i \theta_{1}}\left(\hat{z}+i \frac{\hat{y}-\hat{x}}{\sqrt{2}}\right), \\
& \mathbf{n}_{2}^{\mathrm{BCC}}=\frac{n_{H}}{2} e^{i \theta_{2}}\left(\hat{z}+i \frac{\hat{y}+\hat{x}}{\sqrt{2}}\right), \\
& \mathbf{n}_{3}^{\mathrm{BCC}}=\frac{n_{H}}{2} e^{i \theta_{3}}\left(\hat{y}+i \frac{\hat{x}-\hat{z}}{\sqrt{2}}\right), \\
& \mathbf{n}_{4}^{\mathrm{BCC}}=\frac{n_{H}}{2} e^{i \theta_{4}}\left(\hat{y}+i \frac{\hat{x}+\hat{z}}{\sqrt{2}}\right), \\
& \mathbf{n}_{5}^{\mathrm{BCC}}=\frac{n_{H}}{2} e^{i \theta_{5}}\left(\hat{x}+i \frac{\hat{z}-\hat{y}}{\sqrt{2}}\right), \\
& \mathbf{n}_{6}^{\mathrm{BCC}}=\frac{n_{H}}{2} e^{i \theta_{6}}\left(\hat{x}+i \frac{\hat{z}+\hat{y}}{\sqrt{2}}\right) .
\end{aligned}
$$

The last three multiple-spin configurations (SC, FCC, 
BCC) were first considered by Binz et al. ${ }^{18}$ under the general effort to understand the "partial-order" phase of bulk MnSi at high pressure ${ }^{20}$. Their method of analyzing the interaction effects is different from ours, and the magnetic field effect was not extensively discussed in their work.

By feeding all eight spin configurations into the free energy form (4) it becomes possible to evaluate their energies as a function of $B$. The quadratic theory consisting of $J, D$, and $\mathbf{B}$ alone were already shown to give degener- ate energy, Eq. (7). Evaluation of the various anisotropy energies can be found in the Appendix and here we only quote the final expression. The final energies are conveniently expressed in the reduced units,

$$
\frac{C}{J} \rightarrow C, \frac{U}{D^{2} / J} \rightarrow U, \frac{W}{D^{2} / J} \rightarrow W, \frac{B}{D^{2} / J} \rightarrow B
$$

$$
\begin{aligned}
& \mathrm{H}[111]: \quad \frac{1}{2} \frac{n_{0}^{2}-1}{1+C / 3}+U\left(-\frac{4}{3} n_{0}^{4}+\frac{4}{3} n_{0}^{2}+1\right)+W\left(-\frac{1}{2} n_{0}^{4}+n_{0}^{2}+\frac{1}{2}\right)-B n_{0}, \\
& \mathrm{H}[110]: \quad \frac{1}{2} \frac{n_{0}^{2}-1}{1+C / 4}+U\left(-2 n_{0}^{4}+2 n_{0}^{2}+1\right)+W\left(-\frac{23}{16} n_{0}^{4}+\frac{15}{8} n_{0}^{2}+\frac{9}{16}\right)-B n_{0}, \\
& \text { Conical : } \quad \frac{1}{2}\left(n_{0}^{2}-1\right)+U+W\left(\frac{7}{4} n_{0}^{4}-\frac{3}{2} n_{0}^{2}+\frac{3}{4}\right)-B n_{0}, \\
& \mathrm{SkX} 2: \quad \frac{1}{2}\left(n_{0}^{2}-1\right)+U\left(-\frac{7}{4} n_{0}^{4}+\frac{3}{2} n_{0}^{2}+\frac{5}{4}\right)+W\left(-\frac{5}{4} n_{0}^{4}+\frac{3}{2} n_{0}^{2}+\frac{3}{4}\right)-B n_{0} \\
& \mathrm{SkX} 3: \quad \frac{1}{2} \frac{n_{0}^{2}-1}{1+C / 8}+U\left(-9 n_{0}\left(\frac{1-n_{0}^{2}}{3}\right)^{3 / 2}-\frac{19}{12} n_{0}^{4}+\frac{7}{6} n_{0}^{2}+\frac{17}{12}\right) \\
& +W\left(-6 n_{0}\left(\frac{1-n_{0}^{2}}{3}\right)^{3 / 2}-\frac{35}{32} n_{0}^{4}+\frac{19}{16} n_{0}^{2}+\frac{29}{32}\right)-B n_{0}, \\
& \mathrm{SC}: \quad \frac{1}{2}\left(n_{0}^{2}-1\right)+U\left(-n_{0}^{4}+\frac{2}{3} n_{0}^{2}+\frac{4}{3}\right)+W\left(-\frac{1}{4} n_{0}^{4}+\frac{1}{2} n_{0}^{2}+\frac{3}{4}\right)-B n_{0}, \\
& \mathrm{FCC}: \quad \frac{1}{2} \frac{n_{0}^{2}-1}{1+C / 3}+U\left(-\frac{11}{12} n_{0}^{4}-\frac{1}{2} n_{0}^{2}+\frac{17}{12}\right)+W\left(-\frac{1}{8} n_{0}^{4}+\frac{1}{4} n_{0}^{2}+\frac{7}{8}\right)-B n_{0}, \\
& \mathrm{BCC}: \quad \frac{1}{2} \frac{n_{0}^{2}-1}{1+C / 8}+U\left(\frac{1}{6} n_{0}^{4}-\frac{2}{3} n_{0}^{2}+\frac{3}{2}\right)+W\left(-\frac{3}{32} n_{0}^{4}+\frac{3}{32} n_{0}^{2}+\frac{29}{32}\right) \\
& -4 \sqrt{27 U^{2}+30 U W+9 W^{2}} n_{0}\left(\frac{1-n_{0}^{2}}{6}\right)^{3 / 2}-B n_{0} .
\end{aligned}
$$

The normalization $n_{0}^{2}+N n_{H}^{2}=1$ is used to express the energies solely in terms of $n_{0}$. The above energies can be minimized for $n_{0}$ between 0 and 1 for a given field strength $B$, and such energies can be compared among the eight candidate states. In particular we are interested in the parameter regime $(C, U, W)$ in which one of the multiple-spiral states becomes the ground state, without invoking the finite-temperature effects as in previous Ginzburg-Landau calculations ${ }^{6,16,17}$.

\section{GROUND STATE PHASES}

Previous two-dimensional lattice model study yielded a phase diagram with the ground states $\mathrm{H}[110]$, SkX2, and SkX3 appearing over various sectors of the $(C, B)$ plane $^{14}$ (The parameter $A_{2}$ in Ref. 14 corresponds to $C$ in the present model). Our energy calculation in the continuum model reproduces qualitatively the same phase diagram (Fig. 2) when the search for minimum energy 


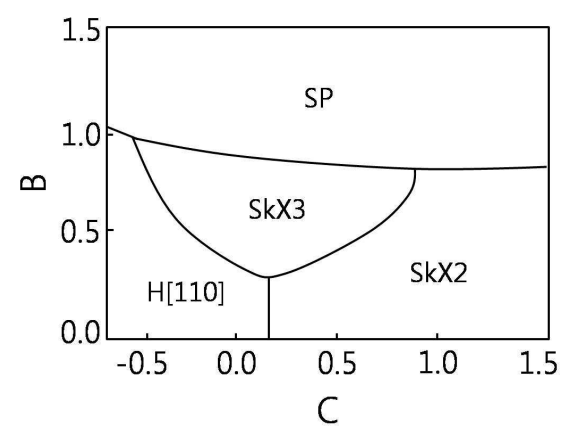

FIG. 2: Ground state phase diagram for two-dimensional spin states, $\{\mathrm{H}[110], \mathrm{SkX} 2, \mathrm{SkX} 3\}$. In a previous work ${ }^{14}$ only $C>0$ was considered. Interaction parameters are $(U, W)=(0,0.1)$ for this phase diagram. SP refers to full spin polarization, or ferromagnetic state.

was confined to three candidate states of two-dimensional character, $\{\mathrm{H}[110], \mathrm{SkX} 2, \mathrm{SkX} 3\}$.
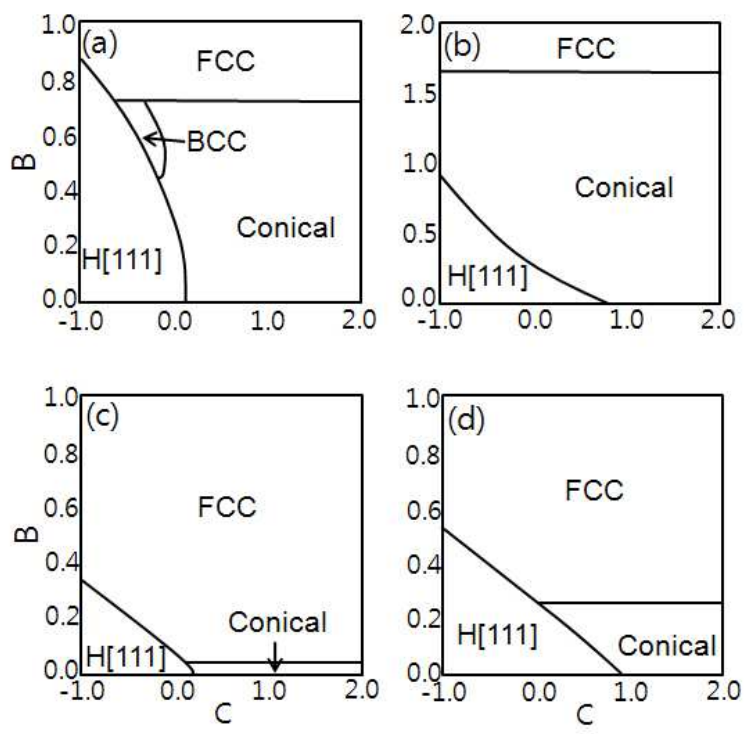

FIG. 3: Ground state phase diagram for all the states we considered. (a) $(U, W)=(0.1,0.1)$, (b) $(U, W)=(0.1,0.5)$, (a) $(U, W)=(0.5,0.1)$, (a) $(U, W)=(0.5,0.5)$.

When three single spiral states are compared in energy, either $\mathrm{H}[111]$ or conical states win out, leaving no room for $\mathrm{H}[110]$ ground state for any of the parameter ranges we searched. We conclude that $\mathrm{H}[110]$ found in the thin-film chiral magnet ${ }^{9}$ was a special feature of two dimensionality.

Now all the states considered in this paper are compared together and the resulting phase diagram is shown in Fig. 3. SkX2 and SkX3 states have disappeared, replaced by spiral states $\mathrm{H}[111]$ or conical at low field. Besides the $(U, W)$ values shown, we have searched for a wider set of parameters but still did not find a region with SkX2, SkX3, or SC ground state. Comparing Fig. 2 and
Fig. 3, we conclude that the two-dimensional Skyrme crystal phases are stable only when the corresponding spatial dimensionality is also two. As shown in Fig. 3, FCC state enjoys a particular energetic advantage in the high-field region while the low-field side is dominated by spiral states. A larger $U$ value tends to push the FCC region down to a lower magnetic field [Fig. 3 (c) and (d)].

\section{SKYRMIONS AND HEDGEHOGS}

In the previous section we have established the phase diagram of the soft-spin model in the variational space of single and multiple spiral phases. The main interest of this paper, we emphasize, is in the identification of threedimensional phases carrying a non-trivial Skyrmion number. A general consideration relates the Skyrmion number to the effective magnetic field felt by the underlying conduction electrons and the emergence of anomalous Hall effect (sometimes called topological Hall effect) ${ }^{21}$. With a three-dimensional space one can define such number for each $x y$ plane along the $z$-axis, the direction of the applied field, as

$$
Q(z)=\frac{1}{4 \pi} \int d^{2} \mathbf{r} \mathbf{n} \cdot\left(\frac{\partial \mathbf{n}}{\partial x} \times \frac{\partial \mathbf{n}}{\partial y}\right) .
$$

The integration extends over one unit cell in real space in the $x y$ plane. This quantity is an integer ${ }^{2}$ for the unitmodulus field satisfying $\left(\mathbf{n}_{\mathbf{r}}\right)^{2}=1$. Our soft-spin configurations generally do not obey this condition locally, but only on average, $\left\langle\mathbf{n}_{\mathbf{r}}^{2}\right\rangle=1$. Let's therefore improve upon the above definition by using the normalized vector field $\hat{\mathbf{n}}=\mathbf{n} /|\mathbf{n}|$ to define the Skyrmion number with proper topological characteristics as

$$
\begin{aligned}
\hat{Q}(z)= & \frac{1}{4 \pi} \int d^{2} \mathbf{r} \hat{\mathbf{n}} \cdot\left(\frac{\partial \hat{\mathbf{n}}}{\partial x} \times \frac{\partial \hat{\mathbf{n}}}{\partial y}\right) \\
& =\frac{1}{4 \pi} \int d^{2} \mathbf{r} \frac{1}{\mathbf{n}^{3}} \mathbf{n} \cdot\left(\frac{\partial \mathbf{n}}{\partial x} \times \frac{\partial \mathbf{n}}{\partial y}\right) .
\end{aligned}
$$

The second line follows from the first by a simple manipulation. In the following, we will be largely concerned with the evaluation of $\hat{Q}(z)$ in each of the multiple-spiral phases and how it depends on the applied field through the uniform magnetization $\mathbf{n}_{0}$.

\section{A. SkX2 \& SkX3}

For the two-dimensional spin crystal SkX2 the spin configuration, up to an overall constant, is given by

$$
\mathbf{n} \propto(\sin y, \cos x, \cos y+\sin x+m) .
$$

We choose $k \equiv 1$ and $m \equiv n_{0} / n_{H}$ for convenience. Actually one finds a periodic array of "nodes" (localized points 
where the magnetization vector $\mathbf{n}$ vanishes) for $m=0$. Expansion around a particular such node $\left(x_{0}, y_{0}\right)$ gives the linearized spin structure

$$
\mathbf{n} \propto\left(y \cos y_{0},-x \sin x_{0}, m-y \cos y_{0}+x \sin x_{0}\right) .
$$

The coordinates are measured from the nodal position. It leads to the Skyrmion density

$$
\frac{1}{4 \pi} \frac{P(m)}{\left[X^{2}+Y^{2}+P(m)^{2}\right]^{3 / 2}},
$$

where

$$
P(m)=\frac{m \sin x_{0} \cos y_{0}}{\sqrt{1-\cos ^{2} x_{0} \sin ^{2} y_{0}}}
$$

and $(X, Y)$ is a linear coordinate transformation of $(x, y)$. The node positions $\left(x_{0}, y_{0}\right)$ are $(-\pi / 2,0)$ and $(\pi / 2, \pi)$ in a unit cell of size $(2 \pi) \times(2 \pi)$, and both points give $P(m)=-m$. Integrating Eq. (22) over $(X, Y)$ gives the Skyrmion number

$$
\hat{Q}(m)=-\frac{1}{2} \operatorname{sgn}[m] .
$$

As $m$ passes from positive to negative, the Skyrmion number changes from $-1 / 2$ to $+1 / 2$. With two such nodes per unit cell, the total Skyrmion number per unit cell depends on $m$ as $-\operatorname{sgn}(m)$. It is convenient to view the situation in the enlarged three-dimensional space consisting of two spatial coordinates $(x, y)$ and one parameter $m$. The set of coordinates $(x, y, m)=\left(x_{0}, y_{0}, 0\right)$, where $\left(x_{0}, y_{0}\right)$ is the node position of SkX2 at $m=0$, define the centers of "hedgehogs". As is well known, a passage through the hedgehog center changes the Skyrmion number by one unit.

One can carry out a similar analysis for SkX3, for which the spin structure reads

$$
\begin{aligned}
\mathbf{n} & \propto\left(n_{x}, n_{y}, n_{z}\right) \\
n_{x} & =\sqrt{3} \cos \frac{x}{2} \sin \frac{\sqrt{3} y}{2} \\
n_{y} & =-\left(2 \cos \frac{x}{2}+\cos \frac{\sqrt{3} y}{2}\right) \sin \frac{x}{2}, \\
n_{z} & =\cos x+2 \cos \frac{x}{2} \cos \frac{\sqrt{3} y}{2}+m .
\end{aligned}
$$

There are no nodes in this magnetization profile, and by direct evaluation one finds $\hat{Q}(z)=-1$ for all uniform magnetization $-1<m<1$. In a sense, $\mathrm{SkX} 3$ is a unique phase carrying a nontrivial Skyrmion number without possessing (or even proximate to) real-space singularities in the spin structure. Indeed, recent experiments ${ }^{9,11}$ single out the SkX3 phase as the only topologically nontrivial magnetic phase so far discovered in chiral magnets.
TABLE I: Nodes of the magnetization vector in a unit cell for SC. $P(z, m)$, defined in Eq. (28), associated with each zero can tell whether a given node is the center of a hedgehog or an an anti-hedgehog $\left(\eta=\sin ^{-1}(m / \sqrt{2})\right)$.

\begin{tabular}{c|c|c|c}
\hline \hline$x_{0}$ & $y_{0}$ & $z_{0}$ & $P(z, m)$ \\
\hline$-\frac{3 \pi}{4}+\eta$ & $\frac{\pi}{4}+\eta$ & $\frac{3 \pi}{4}+\eta$ & $\sqrt{2-m^{2}}\left(1-m^{2}\right) z / 2$ \\
$\frac{3 \pi}{4}+\eta$ & $\frac{3 \pi}{4}+\eta$ & $\frac{3 \pi}{4}-\eta$ & $\sqrt{2-m^{2}}\left(m^{2}-1\right) z / 2$ \\
$\frac{3 \pi}{4}+\eta$ & $-\frac{3 \pi}{4}-\eta$ & $\frac{\pi}{4}+\eta$ & $\sqrt{2-m^{2}}\left(1-m^{2}\right) z / 2$ \\
$-\frac{3 \pi}{4}+\eta$ & $-\frac{\pi}{4}-\eta$ & $\frac{\pi}{4}-\eta$ & $\sqrt{2-m^{2}}\left(m^{2}-1\right) z / 2$ \\
$-\frac{\pi}{4}-\eta$ & $-\frac{\pi}{4}-\eta$ & $-\frac{\pi}{4}+\eta$ & $\sqrt{2-m^{2}}\left(1-m^{2}\right) z / 2$ \\
$\frac{\pi}{4}-\eta$ & $-\frac{3 \pi}{4}-\eta$ & $-\frac{\pi}{4}-\eta$ & $\sqrt{2-m^{2}}\left(m^{2}-1\right) z / 2$ \\
$\frac{\pi}{4}-\eta$ & $\frac{3 \pi}{4}+\eta$ & $-\frac{3 \pi}{4}+\eta$ & $\sqrt{2-m^{2}}\left(1-m^{2}\right) z / 2$ \\
$-\frac{\pi}{4}-\eta$ & $\frac{\pi}{4}+\eta$ & $-\frac{3 \pi}{4}-\eta$ & $\sqrt{2-m^{2}}\left(m^{2}-1\right) z / 2$ \\
\hline \hline
\end{tabular}

One easily realizes that a node in the magnetization is a rare event in the two-dimensional spin structure. Each component of the spin vector taken equal to zero define a two-dimensional surface embedded in a threedimensional space. For 2D spin structures such as SkX2 and SkX3, such a surface is extended vertically along the $z$-axis and traces out a curve in the $x y$ plane. The interaction of three curves, from each component of the spin vector taken to zero, defines the node. The three curves in a plane do not cross at a single point except through fine tuning, such as the tuning of $n_{0}$ through zero in the SkX2 case. With both SkX2 and SkX3, the nodeless spin structures also happen to be topologically nontrivial and carry a finite Skyrmion number.

The same reasoning applied to a generic threedimensional spin crystal structure states that nodes are unavoidable because three independent surfaces would cross at isolated points. Below we show that each such node is the center of a hedgehog or an anti-hedgehog, a passage through which the Skyrmion number changes by $+1(-1)$.

\section{B. SC, FCC \& BCC}

Taking $k=1$, the spin configuration for SC becomes

$$
\mathbf{n} \propto(\sin y+\cos z, \sin z+\cos x, \sin x+\cos y+m) .
$$

The three phase factors $\theta_{\alpha}$ are all assumed to be zero. There are eight independent zeroes in a unit cell and their coordinates are listed in Table I. Taylor-expanding around a given node $\left(x_{0}, y_{0}, z_{0}\right)$ to first order in the deviation results in

$\mathbf{n} \propto\left(y \cos y_{0}-z \sin z_{0}, z \cos z_{0}-x \sin x_{0}, x \cos x_{0}-y \sin y_{0}\right)$. 
The coordinates $(x, y, z)$ are measured from their zeroes. The triple product $\mathbf{n} \cdot \partial_{x} \mathbf{n} \times \partial_{y} \mathbf{n}=z\left(\cos x_{0} \cos y_{0} \cos z_{0}-\right.$ $\left.\sin x_{0} \sin y_{0} \sin z_{0}\right)$ follows immediately, and the normalized Skyrmion density becomes

$$
\frac{1}{4 \pi} \frac{P(z, m)}{\left[X^{2}+Y^{2}+P(z, m)^{2}\right]^{3 / 2}}
$$

where

$$
P(z, m)=\frac{z\left(\cos x_{0} \cos y_{0} \cos z_{0}-\sin x_{0} \sin y_{0} \sin z_{0}\right)}{\sqrt{1-\cos ^{2} x_{0} \sin ^{2} y_{0}}},
$$

and $(X, Y)$ is again related to $(x, y)$ through linear coordinate change. Integral over $(X, Y)$ yields the Skyrmion number

$$
\hat{Q}(z)=\frac{1}{2} \operatorname{sgn}[P(z, m)],
$$

with $P(z, m)$ for each node listed in Table I. We define such node with $\hat{Q}\left(z=0^{+}\right)-\hat{Q}\left(z=0^{-}\right)=+1$ as the hedgehog center, and the other one with $\hat{Q}(z=$ $\left.0^{+}\right)-\hat{Q}\left(z=0^{-}\right)=-1$ as the anti-hedgehog center. A snapshot of a pair of hedgehog/anti-hedgehog configurations obtained from Eq. (27) is shown in Fig. 4.

As can be seen from Table I, hedgehogs and antihedgehogs occur in alternating fashion along the $z$-axis. Average over a unit cell in the $z$-direction yields the average Skyrmion number $\bar{Q}=\int_{0}^{2 \pi} d z \hat{Q}(z) / 2 \pi$ which equals $\left(\eta \equiv \sin ^{-1}(m / \sqrt{2})\right.$

$$
\begin{aligned}
& \bar{Q}=\left\{\begin{array}{ll}
-\frac{4}{\pi} \eta & \left(0<\eta<\frac{\pi}{4}\right) \\
\frac{4}{\pi}\left(\eta-\frac{\pi}{2}\right) & \left(\frac{\pi}{4}<\eta<\frac{\pi}{2}\right)
\end{array}\right\} \\
& \eta \equiv \sin ^{-1}(m / \sqrt{2}) .
\end{aligned}
$$

When $m$ exceeds $\sqrt{2}, \hat{Q}(z)$ is identically zero.
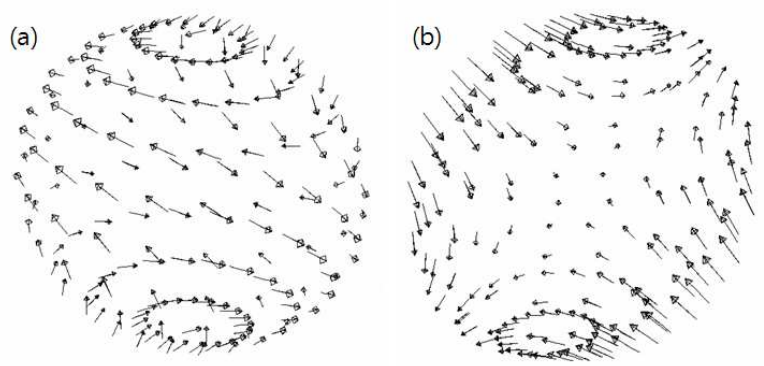

FIG. 4: Spin configuration around (a) hedgehog and (b) antihedgehog center in the SC spin crystal. Magnetization vector $\mathbf{n}$ vanishes at the center of each singularity.

The FCC spin configuration can be analyzed with the similar strategy after taking $k=\sqrt{3}$. Keeping track of the node positions proved difficult for general phase angles, but we do achieve some simplification with special choices, e.g. $\theta_{1}=\theta_{2}=0$ and $\theta_{3}=-\theta_{4}=-\pi / 3$. In this case nodes occurs at

$$
\begin{aligned}
\cos z(\cos (x+y)-\sqrt{3} \sin (x-y)) & =0, \\
\sin z(\sqrt{3} \cos (x+y)-\sin (x-y)) & =0, \\
2 \sin z \sin (x+y)+2 \cos z \cos (x-y)+m & =0 .
\end{aligned}
$$

One possible set of zeroes come from choosing $\cos z=0$, $\sqrt{3} \cos (x+y)=\sin (x-y)$ and $2 \sin z \sin (x+y)+m=$ 0 . There are eight possible solutions. Another possible set is $\sin z=0, \cos (x+y)=\sqrt{3} \sin (x-y)$ and $2 \cos z \cos (x-y)+m=0$. This gives another eight zeroes. A third set arises from having $\cos (x+y)=0, \sin (x-y)=0$ and $\sin z(\sqrt{3} \cos (x+y)-\sin (x-y))=0$. There are 16 independent nodes that belong to the third set as listed in Table II. From the Table one can read off the $z$-integrated Skyrmion number. The result is in fact twice that given Eq. (30) for SC if one replaces $\eta=\sin ^{-1}(m / \sqrt{2})$ with $\xi=\sin ^{-1}(m / 2 \sqrt{2})$ now. The first two sets of nodes contribute zero Skyrmion number as each $z$-plane contains an equal number of hedgehogs and anti-hedgehogs. For BCC spin crystal phase Binz and Vishwanath obtained $\bar{Q}=-\operatorname{sgn}(m)(2 / \pi) \cos ^{-1}(|m| / 2 \sqrt{2})$ for $|m|<2 \sqrt{2}$ and zero otherwise $^{22}$. Together with our calculation, a complete information is available for the magnetization (magnetic field dependence) of the Skyrmion number for bulk spin crystal phases.

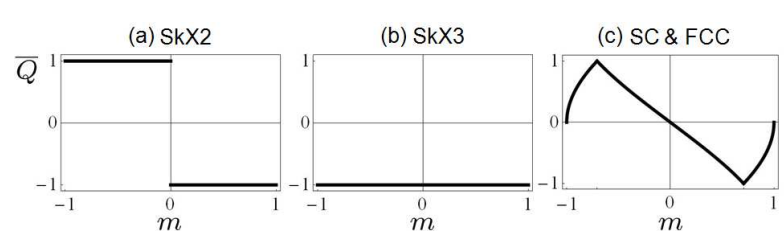

FIG. 5: $z$-integrated Skyrmion number $\bar{Q}$ for (a) SkX2, (b) SkX3, and (c) SC and FCC spin crystals. The FCC result is multiplied by $1 / 2$ in the figure. Magnetization $m$ is normalized to unity at the maximum value with nonzero $\bar{Q}$.

\section{Nature of 3D Spin Crystals}

Based on the analysis of the multiple-spiral states both of $2 \mathrm{D}$ and $3 \mathrm{D}$ characters, we are led to suspect that realizing a topologically non-trivial (in the sense of having nonzero $\hat{Q}(z))$ spin structure free of singularities is extremely difficult in three dimensions, whereas it should be quite easy in two dimensions. A genuine 3D Skyrmion of course is without a singularity ${ }^{23}$, and only a lattice of 
TABLE II: Nodes of the magnetization vector in a unit cell for $\left.\operatorname{FCC}\left(\xi=\sin ^{-1}(m / 2 \sqrt{2})\right)\right)$.

\begin{tabular}{c|c|c|c}
\hline \hline$x_{0}$ & $y_{0}$ & $z_{0}$ & \multicolumn{1}{c}{$P(z, m)$} \\
\hline$\pi / 4$ & $5 \pi / 4$ & $7 \pi / 4+\xi$ & $\sqrt{8-m^{2}}\left(4-m^{2}\right) z$ \\
$5 \pi / 4$ & $\pi / 4$ & $7 \pi / 4+\xi$ & $\sqrt{8-m^{2}}\left(4-m^{2}\right) z$ \\
$\pi / 4$ & $\pi / 4$ & $7 \pi / 4-\xi$ & $\sqrt{8-m^{2}}\left(m^{2}-4\right) z$ \\
$5 \pi / 4$ & $5 \pi / 4$ & $7 \pi / 4-\xi$ & $\sqrt{8-m^{2}}\left(m^{2}-4\right) z$ \\
$3 \pi / 4$ & $7 \pi / 4$ & $5 \pi / 4+\xi$ & $\sqrt{8-m^{2}}\left(4-m^{2}\right) z$ \\
$7 \pi / 4$ & $3 \pi / 4$ & $5 \pi / 4+\xi$ & $\sqrt{8-m^{2}}\left(4-m^{2}\right) z$ \\
$3 \pi / 4$ & $3 \pi / 4$ & $5 \pi / 4-\xi$ & $\sqrt{8-m^{2}}\left(m^{2}-4\right) z$ \\
$7 \pi / 4$ & $7 \pi / 4$ & $5 \pi / 4-\xi$ & $\sqrt{8-m^{2}}\left(m^{2}-4\right) z$ \\
$\pi / 4$ & $\pi / 4$ & $3 \pi / 4+\xi$ & $\sqrt{8-m^{2}}\left(4-m^{2}\right) z$ \\
$5 \pi / 4$ & $5 \pi / 4$ & $3 \pi / 4+\xi$ & $\sqrt{8-m^{2}}\left(4-m^{2}\right) z$ \\
$\pi / 4$ & $5 \pi / 4$ & $3 \pi / 4-\xi$ & $\sqrt{8-m^{2}}\left(m^{2}-4\right) z$ \\
$5 \pi / 4$ & $\pi / 4$ & $3 \pi / 4-\xi$ & $\sqrt{8-m^{2}}\left(m^{2}-4\right) z$ \\
$3 \pi / 4$ & $3 \pi / 4$ & $\pi / 4+\xi$ & $\sqrt{8-m^{2}}\left(4-m^{2}\right) z$ \\
$7 \pi / 4$ & $7 \pi / 4$ & $\pi / 4+\xi$ & $\sqrt{8-m^{2}}\left(4-m^{2}\right) z$ \\
$3 \pi / 4$ & $7 \pi / 4$ & $\pi / 4-\xi$ & $\sqrt{8-m^{2}}\left(m^{2}-4\right) z$ \\
$7 \pi / 4$ & $3 \pi / 4$ & $\pi / 4-\xi$ & $\sqrt{8-m^{2}}\left(m^{2}-4\right) z$ \\
\hline \hline
\end{tabular}

such structures would qualify as a "3D Skymre crystal" 24 . All the 3D states discussed in this paper and elsewhere ${ }^{18}$ can be instead described as a "hedgehog crystal".

In the hard-spin limit where $\mathbf{n}^{2}=1$ is rigorously enforced at the local level, we will find that a periodic array of singularities becomes energetically forbidding and give way to single-spiral structures without nodes. It is possible, however, that the spins remain soft in real chiral magnets due to interaction with the metallic host that can "absorb" some of the spin from the local moments ${ }^{16}$. In such a case, the singularities inherent in the hedgehog lattice can be realized without an excessive cost in energy. The other exciting possibility is that the spins remain hard even against the metallic background, and yet somehow form a topologically non-trivial 3D Skyrme crystal phase such as envisioned by Klebanov and others ${ }^{24}$. Whether such a state can be energetically stable compared to hedgehog or single-spiral phases is a subject yet to be explored in the context of chiral magnetism. Creating a singularity-free Skyrmion lattice structure requires the inclusion of higher-order harmonics (in the sense of Fourier decomposition of the spin structure), which also partly explains why our first-harmonic construction only yields hedgehog lattices.

\section{SUMMARY}

The ground state energies of several single- and multiple-spiral spin configurations are considered, on the basis of the Ginzburg-Landau free energy suggested by
Bak and Jensen ${ }^{19}$. Phase diagrams in the space of anisotropy and magnetic field $(C, B)$ are worked out for a variety of interaction parameters $(U, W)$. Previous two-dimensional phase diagram ${ }^{14}$ is recovered and threedimensional phase diagrams are worked out anew. Similar efforts were made earlier by Binz et al. ${ }^{18}$ to carve out the 3D phase diagram, focusing on the stability of $3 \mathrm{D}$ spin textures such as SC, FCC, and BCC, under zero magnetic field and using the interaction terms which are non-local in space. Leonov et al. ${ }^{17}$ considered the phase diagram spanned by temperature and field, with twodimensional spin textures SkX2 and SkX3 as candidate states. Our search is confined to zero-temperature, but covers both 2D and 3D spin textures.

As shown in Fig. 3, magnetic field plays a crucial role in stabilizing Skyrmionic order over single-spiral states in both 2D and 3D geometries. Generically, twodimensional Skyrme crystals (SkX2 and SkX3) are favored only if the corresponding effective space dimensionality is two. For three dimensions, 3D Skyrmion structures such as FCC and BCC are more favorable energetically. Our calculation suggests the possibility for a spin crystal phase of FCC symmetry very close to zero magnetic field for a sufficiently strong interaction parameter $U$.

The Skyrmion number embodied by each multiple spiral phase was examined. An array of hedgehog and antihedgehog centers were identified in the case of threedimensional spin crystals. Passage through each such center change the Skyrmion number by \pm 1 . Generally there exists a non-zero, $z$-integrated Skyrmion number for finite uniform magnetization. We showed that SkX3 is the only spin crystal structure without singularities and yet exhibiting a nonzero Skyrmion number. Constructing a singularity-free spin crystal state in three-dimensional chiral magnet is an interesting challenge for the future.

\section{Acknowledgments}

H. J. H. is supported by Mid-career Researcher Program through NRF grant funded by the MEST (No. R012008-000-20586-0), and in part by the Asia Pacific Center for Theoretical Physics. H. J. H. is grateful to Naoto Nagaosa and Jan Zaanen for their invaluable inputs on Skyrmions and hedgehogs.

\section{Appendix A: Calculation of $C$-anisotropy}

To calculate the $C$-anisotropy one should first obtain $\partial n_{x} / \partial x$ :

$$
\begin{aligned}
\frac{\partial n_{x}}{\partial x} & =\sum_{\alpha}\left(i k_{\alpha}^{x} n_{\alpha}^{x} e^{i \mathbf{k}_{\alpha} \cdot \mathbf{r}}-i k_{\alpha}^{x}\left(n_{\alpha}^{x}\right)^{*} e^{-i \mathbf{k}_{\alpha} \cdot \mathbf{r}}\right) \\
\int\left(\frac{\partial n_{x}}{\partial x}\right)^{2} & =2 \sum_{\alpha}\left(k_{\alpha}^{x}\right)^{2} n_{\alpha}^{x}\left(n_{\alpha}^{x}\right)^{*} .
\end{aligned}
$$


With similar expressions from $\int\left(\partial n_{y} / \partial y\right)^{2}$ and $\int\left(\partial n_{z} / \partial z\right)^{2}, C$-anisotropy energy reads

$$
\begin{aligned}
& \frac{1}{2} C \int\left[\left(\frac{\partial n_{x}}{\partial x}\right)^{2}+\left(\frac{\partial n_{y}}{\partial y}\right)^{2}+\left(\frac{\partial n_{z}}{\partial z}\right)^{2}\right]= \\
& C \sum_{\alpha}\left(\left(k_{\alpha}^{x}\right)^{2} n_{\alpha}^{x}\left(n_{\alpha}^{x}\right)^{*}+\left(k_{\alpha}^{y}\right)^{2} n_{\alpha}^{y}\left(n_{\alpha}^{y}\right)^{*}+\left(k_{\alpha}^{z}\right)^{2} n_{\alpha}^{z}\left(n_{\alpha}^{z}\right)^{*}\right) .
\end{aligned}
$$

The eight states have the $C$-anisotropy energies

$$
\begin{aligned}
\text { Helical }\left(\mathrm{H}_{111}\right): & (1 / 6) C n_{H}^{2} k^{2} \\
\text { Helical }\left(\mathrm{H}_{110}\right): & (1 / 8) C n_{H}^{2} k^{2} \\
\text { Conical : } & 0 \\
\text { SkX2 : } & 0 \\
\text { SkX3 : } & (3 / 16) C n_{H}^{2} k^{2} \\
\mathrm{SC}: & 0 \\
\mathrm{FCC}: & (2 / 3) C n_{H}^{2} k^{2} \\
\mathrm{BCC}: & (3 / 8) C n_{H}^{2} k^{2}
\end{aligned}
$$

Including the $C$-anisotropy gives the total energy for the various spin states

$$
\begin{aligned}
\mathrm{H}_{111}: & \frac{1}{2} n_{H}^{2}\left(J k^{2}-2 D k\right)+\frac{1}{6} C n_{H}^{2} k^{2} \\
\mathrm{H}_{110}: & \frac{1}{2} n_{H}^{2}\left(J k^{2}-2 D k\right)+\frac{1}{8} C n_{H}^{2} k^{2} \\
\mathrm{Co}: & \frac{1}{2} n_{H}^{2}\left(J k^{2}-2 D k\right) \\
\mathrm{SkX} 2: & n_{H}^{2}\left(J k^{2}-2 D k\right) \\
\mathrm{SkX} 3: & \frac{3}{2} n_{H}^{2}\left(J k^{2}-2 D k\right)+\frac{3}{16} C n_{H}^{2} k^{2} \\
\mathrm{SC}: & \frac{3}{2} n_{H}^{2}\left(J k^{2}-2 D k\right) \\
\mathrm{FCC}: & 2 n_{H}^{2}\left(J k^{2}-2 D k\right)+\frac{2}{3} C n_{H}^{2} k^{2} \\
\mathrm{BCC}: & 3 n_{H}^{2}\left(J k^{2}-2 D k\right)+\frac{3}{8} C n_{H}^{2} k^{2}
\end{aligned}
$$

As the remaining $U$ and $W$ interactions do not contain the $k$-dependence, one can minimize $k$ for the above energies and obtain the optimal $\mathbf{k}$-vector length for each spin state

$$
\begin{array}{rlr}
k^{\mathrm{H}_{111}}=D /(J+C / 3), & k^{\mathrm{H}_{110}}=D /(J+C / 4), \\
k^{\mathrm{Co}}=D / J, & k^{\mathrm{SkX} 2}=D / J, \\
k^{\mathrm{SkX} 3}=D /(J+C / 8), & k^{\mathrm{SC}}=D / J, \\
k^{\mathrm{FCC}}=D /(J+C / 3), & k^{\mathrm{BCC}}=D /(J+C / 8) .
\end{array}
$$

\section{Appendix B: Calculation of $U$-energy}

Dividing up the spin configuration $\mathbf{n}=\mathbf{n}_{0}+\boldsymbol{\Phi}$ as in Eq. (1), $\mathbf{n}^{4}$ is expanded as

$$
\begin{aligned}
\mathbf{n}^{4}= & \left(\mathbf{n}_{0}^{2}+2 \mathbf{n}_{0} \cdot \boldsymbol{\Phi}+\boldsymbol{\Phi}^{2}\right)^{2} \\
= & \mathbf{n}_{0}^{4}+4\left(\mathbf{n}_{0} \cdot \boldsymbol{\Phi}\right)^{2}+4 \mathbf{n}_{0}^{2}\left(\mathbf{n}_{0} \cdot \boldsymbol{\Phi}\right) \\
& +4 \boldsymbol{\Phi}^{2}\left(\mathbf{n}_{0} \cdot \boldsymbol{\Phi}\right)+2 \mathbf{n}_{0}^{2} \boldsymbol{\Phi}^{2}+\boldsymbol{\Phi}^{4} .
\end{aligned}
$$

$\mathrm{H}[111]$ state gives $\int\left(\mathbf{n}_{0} \cdot \boldsymbol{\Phi}\right)^{2}=n_{0}^{2} n_{H}^{2} / 3, \int \boldsymbol{\Phi}^{2}=n_{H}^{2}$, $\int \boldsymbol{\Phi}^{4}=n_{H}^{4}$, and the $U$-energy

$$
\mathrm{H}[111]: U\left(n_{0}^{4}+\frac{10}{3} n_{0}^{2} n_{H}^{2}+n_{H}^{4}\right) .
$$

$\mathrm{H}[110]$ state gives $\int\left(\mathbf{n}_{0} \cdot \boldsymbol{\Phi}\right)^{2}=n_{0}^{2} n_{H}^{2} / 2, \int \boldsymbol{\Phi}^{2}=n_{H}^{2}$, $\int \boldsymbol{\Phi}^{4}=n_{H}^{4}$, and the $U$-energy

$$
\mathrm{H}[110]: U\left(n_{0}^{4}+4 n_{0}^{2} n_{H}^{2}+n_{H}^{4}\right) .
$$

Conical state gives $\int\left(\mathbf{n}_{0} \cdot \boldsymbol{\Phi}\right)^{2}=0, \int \boldsymbol{\Phi}^{2}=n_{H}^{2}, \int \boldsymbol{\Phi}^{4}=$ $n_{H}^{4}$, and the $U$-energy

$$
\text { Conical : } U\left(n_{0}^{4}+2 n_{0}^{2} n_{H}^{2}+n_{H}^{4}\right) \text {. }
$$

SkX2 state with $\int\left(\mathbf{n}_{0} \cdot \boldsymbol{\Phi}\right)^{2}=n_{0}^{2} n_{H}^{2}, \int \Phi^{2}=2 n_{H}^{2}$, and $\int \boldsymbol{\Phi}^{4}=5 n_{H}^{4}$ gives the $U$-energy

$$
\mathrm{SkX} 2: U\left(n_{0}^{4}+8 n_{0}^{2} n_{H}^{2}+5 n_{H}^{4}\right) .
$$

There are four types of terms contributing to $\int \boldsymbol{\Phi}^{4}$.

$$
\begin{aligned}
&-\sum_{\alpha}\left(\mathbf{n}_{\alpha} \cdot \mathbf{n}_{\alpha}^{*}\right)\left(\mathbf{n}_{\alpha} \cdot \mathbf{n}_{\alpha}^{*}\right)=2 n_{H}^{4} \\
& \text { - } \sum_{l \neq m}\left(\mathbf{n}_{l} \cdot \mathbf{n}_{l}^{*}\right)\left(\mathbf{n}_{m} \cdot \mathbf{n}_{m}^{*}\right)=2 n_{H}^{4} \\
& \text { - } \sum_{l \neq m}\left(\mathbf{n}_{l} \cdot \mathbf{n}_{m}\right)\left(\mathbf{n}_{l}^{*} \cdot \mathbf{n}_{m}^{*}\right)=(1 / 2) n_{H}^{4} \\
& \text { - } \sum_{l \neq m}\left(\mathbf{n}_{l} \cdot \mathbf{n}_{m}^{*}\right)\left(\mathbf{n}_{l}^{*} \cdot \mathbf{n}_{m}\right)=(1 / 2) n_{H}^{4}
\end{aligned}
$$

SkX3 state gives $\int\left(\mathbf{n}_{0} \cdot \boldsymbol{\Phi}\right)^{2}=(3 / 2) n_{0}^{2} n_{H}^{2}$ and $\int \boldsymbol{\Phi}^{2}\left(\mathbf{n}_{0}\right.$. $\Phi)$ is non-vanishing,

$$
\begin{aligned}
\int \Phi^{2}\left(\mathbf{n}_{0} \cdot \boldsymbol{\Phi}\right) & =\sum_{\mathbf{k}_{1}+\mathbf{k}_{2}+\mathbf{k}_{3}=\mathbf{0}}\left(\mathbf{n}_{0} \cdot \mathbf{n}_{\mathbf{k}_{1}}\right)\left(\mathbf{n}_{\mathbf{k}_{2}} \cdot \mathbf{n}_{\mathbf{k}_{3}}\right) \\
& =9 n_{0} n_{H}^{3} \cos \left(\theta_{1}+\theta_{2}+\theta_{3}\right) .
\end{aligned}
$$

There are 24 combinations of the type

$$
\int\left(\mathbf{n}_{0} \cdot \mathbf{n}_{1}\right)\left(\mathbf{n}_{2} \cdot \mathbf{n}_{3}\right)=\frac{3}{16} n_{0} n_{H}^{3} e^{i\left(\theta_{1}+\theta_{2}+\theta_{3}\right)}
$$

and another 24 from their complex conjugates contributing to $\int \boldsymbol{\Phi}^{2}\left(\mathbf{n}_{0} \cdot \boldsymbol{\Phi}\right)$. $\int \boldsymbol{\Phi}^{4}$ gets contributions from the following four terms.

$$
\text { - } \sum_{\alpha}\left(\mathbf{n}_{\alpha} \cdot \mathbf{n}_{\alpha}^{*}\right)\left(\mathbf{n}_{\alpha} \cdot \mathbf{n}_{\alpha}^{*}\right)=3 n_{H}^{4}
$$


- $\sum_{l \neq m}\left(\mathbf{n}_{l} \cdot \mathbf{n}_{l}^{*}\right)\left(\mathbf{n}_{m} \cdot \mathbf{n}_{m}^{*}\right)=6 n_{H}^{4}$

- $\sum_{l \neq m}\left(\mathbf{n}_{l} \cdot \mathbf{n}_{m}\right)\left(\mathbf{n}_{l}^{*} \cdot \mathbf{n}_{m}^{*}\right)=(27 / 8) n_{H}^{4}$

- $\sum_{l \neq m}\left(\mathbf{n}_{l} \cdot \mathbf{n}_{m}^{*}\right)\left(\mathbf{n}_{l}^{*} \cdot \mathbf{n}_{m}\right)=(3 / 8) n_{H}^{4}$

Overall, SkX3 has the $U$-energy

$$
\begin{array}{r}
\text { SkX3 : } U\left(n_{0}^{4}+9 n_{0} n_{H}^{3} \cos \left(\theta_{1}+\theta_{2}+\theta_{3}\right)\right. \\
\left.+12 n_{0}^{2} n_{H}^{2}+\frac{51}{4} n_{H}^{4}\right) .
\end{array}
$$

The phase angles influence the total energy as $\sim \cos \left(\theta_{1}+\right.$ $\left.\theta_{2}+\theta_{3}\right)$. It can be minimized by choosing $\theta_{1}+\theta_{2}+\theta_{3}=\pi$.

SC state gives $\int\left(\mathbf{n}_{0} \cdot \boldsymbol{\Phi}\right)^{2}=n_{0}^{2} n_{H}^{2}$ and $\int \boldsymbol{\Phi}^{2}=3 n_{H}^{2}$. $\int \boldsymbol{\Phi}^{4}=12 n_{H}^{4}$ is obtained from

- $\sum_{\alpha}\left(\mathbf{n}_{\alpha} \cdot \mathbf{n}_{\alpha}^{*}\right)\left(\mathbf{n}_{\alpha} \cdot \mathbf{n}_{\alpha}^{*}\right)=3 n_{H}^{4}$

- $\sum_{l \neq m}\left(\mathbf{n}_{l} \cdot \mathbf{n}_{l}^{*}\right)\left(\mathbf{n}_{m} \cdot \mathbf{n}_{m}^{*}\right)=6 n_{H}^{4}$

- $\sum_{l \neq m}\left(\mathbf{n}_{l} \cdot \mathbf{n}_{m}\right)\left(\mathbf{n}_{l}^{*} \cdot \mathbf{n}_{m}^{*}\right)=(3 / 2) n_{H}^{4}$

- $\sum_{l \neq m}\left(\mathbf{n}_{l} \cdot \mathbf{n}_{m}^{*}\right)\left(\mathbf{n}_{l}^{*} \cdot \mathbf{n}_{m}\right)=(3 / 2) n_{H}^{4}$

$U$-energy for SC reads

$$
\mathrm{SC}: U\left(n_{0}^{4}+10 n_{0}^{2} n_{H}^{2}+12 n_{H}^{4}\right)
$$

FCC state gives $\int\left(\mathbf{n}_{0} \cdot \boldsymbol{\Phi}\right)^{2}=4 n_{0}^{2} n_{H}^{2} / 3$, and $\int \boldsymbol{\Phi}^{2}=$ $4 n_{H}^{2} \cdot \int \boldsymbol{\Phi}^{4}=(68 / 3) n_{H}^{4}$ derives from

- $\sum_{\alpha}\left(\mathbf{n}_{\alpha} \cdot \mathbf{n}_{\alpha}^{*}\right)\left(\mathbf{n}_{\alpha} \cdot \mathbf{n}_{\alpha}^{*}\right)=4 n_{H}^{4}$

- $\sum_{l \neq m}\left(\mathbf{n}_{l} \cdot \mathbf{n}_{l}^{*}\right)\left(\mathbf{n}_{m} \cdot \mathbf{n}_{m}^{*}\right)=12 n_{H}^{4}$

- $\sum_{l \neq m}\left(\mathbf{n}_{l} \cdot \mathbf{n}_{m}\right)\left(\mathbf{n}_{l}^{*} \cdot \mathbf{n}_{m}^{*}\right)=(8 / 3) n_{H}^{4}$

- $\sum_{l \neq m}\left(\mathbf{n}_{l} \cdot \mathbf{n}_{m}^{*}\right)\left(\mathbf{n}_{l}^{*} \cdot \mathbf{n}_{m}\right)=4 n_{H}^{4}$

A careful calculation shows that contributions from all four wave vectors different give zero contribution to $\int \Phi^{4}$. The $U$-energy reads

$$
\mathrm{FCC}: U\left(n_{0}^{4}+\frac{40}{3} n_{0}^{2} n_{H}^{2}+\frac{68}{3} n_{H}^{4}\right)
$$

BCC state gives $\int\left(\mathbf{n}_{0} \cdot \boldsymbol{\Phi}\right)^{2}=2 n_{0}^{2} n_{H}^{2}$ and $\int \boldsymbol{\Phi}^{2}=6 n_{H}^{2}$. $\int \boldsymbol{\Phi}^{4}=54 n_{H}^{4}$ consists of the following terms.

- $\sum_{\alpha}\left(\mathbf{n}_{\alpha} \cdot \mathbf{n}_{\alpha}^{*}\right)\left(\mathbf{n}_{\alpha} \cdot \mathbf{n}_{\alpha}^{*}\right)=6 n_{H}^{4}$

- $\sum_{l \neq m}\left(\mathbf{n}_{l} \cdot \mathbf{n}_{l}^{*}\right)\left(\mathbf{n}_{m} \cdot \mathbf{n}_{m}^{*}\right)=30 n_{H}^{4}$

- $\sum_{l \neq m}\left(\mathbf{n}_{l} \cdot \mathbf{n}_{m}\right)\left(\mathbf{n}_{l}^{*} \cdot \mathbf{n}_{m}^{*}\right)=9 n_{H}^{4}$

- $\sum_{l \neq m}\left(\mathbf{n}_{l} \cdot \mathbf{n}_{m}^{*}\right)\left(\mathbf{n}_{l}^{*} \cdot \mathbf{n}_{m}\right)=9 n_{H}^{4}$
As in SkX3,

$$
\int \boldsymbol{\Phi}^{2}\left(\mathbf{n}_{0} \cdot \boldsymbol{\Phi}\right)=\sum_{\mathbf{q}_{1}+\mathbf{q}_{2}+\mathbf{q}_{3}=\mathbf{0}}\left(\mathbf{n}_{0} \cdot \mathbf{n}_{\mathbf{q}_{1}}\right)\left(\mathbf{n}_{\mathbf{q}_{2}} \cdot \mathbf{n}_{\mathbf{q}_{3}}\right)
$$

is non-vanishing from combination of vectors $\mathbf{k}_{1}+\mathbf{k}_{4}-\mathbf{k}_{5}=$ $\mathbf{0}, \mathbf{k}_{3}+\mathbf{k}_{6}-\mathbf{k}_{1}=\mathbf{0}, \mathbf{k}_{2}+\mathbf{k}_{5}-\mathbf{k}_{3}=\mathbf{0}, \mathbf{k}_{2}+\mathbf{k}_{4}+\mathbf{k}_{6}=\mathbf{0}$. The $U$-energy becomes

$$
\begin{aligned}
& \mathrm{BCC}: U\left(n_{0}^{4}+14 n_{0}^{2} n_{H}^{2}+54 n_{H}^{4}\right) \\
& \quad+U n_{0} n_{H}^{3}( \\
& 5 \cos \left(\theta_{1}+\theta_{4}-\theta_{5}\right)-\sqrt{2} \sin \left(\theta_{1}+\theta_{4}-\theta_{5}\right) \\
& +5 \cos \left(\theta_{3}+\theta_{6}-\theta_{1}\right)-\sqrt{2} \sin \left(\theta_{3}+\theta_{6}-\theta_{1}\right) \\
& -5 \cos \left(\theta_{2}+\theta_{5}-\theta_{3}\right)+\sqrt{2} \sin \left(\theta_{2}+\theta_{5}-\theta_{3}\right) \\
& \left.-5 \cos \left(\theta_{2}+\theta_{4}+\theta_{6}\right)-\sqrt{2} \sin \left(\theta_{2}+\theta_{4}+\theta_{6}\right)\right) .
\end{aligned}
$$

The phase angle dependence of the $U$-energy is more complicated than in the case of SkX3 because there are six such angles instead of three.

\section{Appendix C: Calculation of $W$-energy}

Spin components are decomposed as $n_{x}=\Phi_{x}, n_{y}=\Phi_{y}$ and $n_{z}=n_{0}+\Phi_{z}$. Hence, $n_{x}^{4}=\Phi_{x}^{4}, n_{y}^{4}=\Phi_{y}^{4}$, and

$$
\begin{aligned}
n_{z}^{4} & =\left(n_{0}^{2}+2 n_{0}^{2} \Phi_{z}^{2}+\Phi_{z}^{2}\right)^{2} \\
& =n_{0}^{4}+6 n_{0}^{2} \Phi_{z}^{2}+4 n_{0}^{3} \Phi_{z}+4 n_{0} \Phi_{z}^{3}+\Phi_{z}^{4}
\end{aligned}
$$

For all the states under consideration we have $\int n_{x}^{4}=$ $\int n_{y}^{4}$.

$\mathrm{H}[111]$ state gives $\int n_{x}^{4}=(1 / 6) n_{H}^{4}$ and $\int n_{z}^{4}=n_{0}^{4}+$ $2 n_{0}^{2} n_{H}^{2}+(1 / 6) n_{H}^{4}$ for the $W$-energy

$$
\mathrm{H}[111]: W\left(n_{0}^{4}+2 n_{0}^{2} n_{H}^{2}+\frac{1}{2} n_{H}^{4}\right) .
$$

$\mathrm{H}[110]$ state gives $\int n_{x}^{4}=(3 / 32) n_{H}^{4}$ and $\int n_{z}^{4}=n_{0}^{4}+$ $3 n_{0}^{2} n_{H}^{2}+(3 / 8) n_{H}^{4}$ for the $W$-energy

$$
\mathrm{H}[110]: W\left(n_{0}^{4}+3 n_{0}^{2} n_{H}^{2}+\frac{9}{16} n_{H}^{4}\right) .
$$

Conical state gives $\int n_{x}^{4}=(3 / 8) n_{H}^{4}$ and $\int n_{z}^{4}=n_{0}^{4}$ for the $W$-energy

$$
\text { Conical : } W\left(\frac{3}{4} n_{H}^{4}+n_{0}^{4}\right)
$$

SkX2 state has two types of terms in calculating $\int \Phi_{z}^{4}=$ $(9 / 4) n_{H}^{4}$. 
- $\sum_{\alpha}\left|\left(n_{\alpha}\right)_{z}\right|^{4}=(3 / 4) n_{H}^{4}$

- $\sum_{l \neq m}\left|\left(n_{l}\right)_{z}\right|^{2}\left|\left(n_{m}\right)_{z}\right|^{2}=(3 / 2) n_{H}^{4}$

With $\int n_{x}^{4}=(3 / 8) n_{H}^{4}$ and $\int n_{z}^{4}=n_{0}^{4}+6 n_{0}^{2} n_{H}^{2}+(9 / 4) n_{H}^{4}$, the $W$-energy becomes

$$
\text { SkX2: } W\left(n_{0}^{4}+6 n_{0}^{2} n_{H}^{2}+3 n_{H}^{4}\right) .
$$

SkX3 state gives

$$
\begin{aligned}
4 n_{0} \int \Phi_{z}^{3} & =6 n_{0}\left(n_{\mathbf{k}_{1}}\right)_{z}\left(n_{\mathbf{k}_{2}}\right)_{z}\left(n_{\mathbf{k}_{3}}\right)_{z} \\
& =6 n_{0} n_{H}^{3} \cos \left(\theta_{1}+\theta_{2}+\theta_{3}\right) .
\end{aligned}
$$

For $\int \Phi_{x}^{4}=\int \Phi_{y}^{4}=(81 / 64) n_{H}^{4}$ one works out

- $\sum_{\alpha}\left|\left(n_{\alpha}\right)_{x}\right|^{4}=(27 / 64) n_{H}^{4}$

- $\sum_{l \neq m}\left|\left(n_{l}\right)_{x}\right|^{2}\left|\left(n_{m}\right)_{x}\right|^{2}=(27 / 32) n_{H}^{4}$

For $\int \Phi_{z}^{4}=(45 / 8) n_{H}^{4}$ one works out

- $\sum_{\alpha}\left|\left(n_{\alpha}\right)_{z}\right|^{4}=(9 / 8) n_{H}^{4}$

- $\sum_{l \neq m}\left|\left(n_{l}\right)_{z}\right|^{2}\left|\left(n_{m}\right)_{z}\right|^{2}=(9 / 2) n_{H}^{4}$

The $W$-energy is $\int n_{x}^{4}=(81 / 64) n_{H}^{4}, \int n_{z}^{4}=n_{0}^{4}+$ $6 n_{0} n_{H}^{3} \cos \left(\theta_{1}+\theta_{2}+\theta_{3}\right)+9 n_{0}^{2} n_{H}^{2}+(45 / 8) n_{H}^{4}$, and

$$
\begin{array}{r}
\text { SkX3 : } W\left(n_{0}^{4}+6 n_{0} n_{H}^{3} \cos \left(\theta_{1}+\theta_{2}+\theta_{3}\right)\right. \\
\left.+9 n_{0}^{2} n_{H}^{2}+\frac{261}{32} n_{H}^{4}\right) .
\end{array}
$$

The phase angle dependence $\sim \cos \left(\theta_{1}+\theta_{2}+\theta_{3}\right)$ arises in the $W$-energy as well as in the $U$-energy for SkX3.

SC state has two types of terms contributing to $\int \Phi_{x}^{4}=$ $\int \Phi_{y}^{4}=\int \Phi_{z}^{4}=(9 / 4) n_{H}^{4}$ :

- $\sum_{\alpha}\left|\left(n_{\alpha}\right)_{z}\right|^{4}=(3 / 4) n_{H}^{4}$

- $\sum_{l \neq m}\left|\left(n_{l}\right)_{z}\right|^{2}\left|\left(n_{m}\right)_{z}\right|^{2}=(3 / 2) n_{H}^{4}$

The $W$-energy is $\int n_{x}^{4}=(9 / 4) n_{H}^{4}, \int n_{z}^{4}=n_{0}^{4}+6 n_{0}^{2} n_{H}^{2}+$ $(9 / 4) n_{H}^{4}$, and

$$
\mathrm{SC}: W\left(n_{0}^{4}+6 n_{0}^{2} n_{H}^{2}+\frac{27}{4} n_{H}^{4}\right)
$$

FCC state has contributions to $\int \Phi_{x}^{4}=\int \Phi_{y}^{4}=\int \Phi_{z}^{4}=$ $(14 / 3) n_{H}^{4}$ coming from

- $\sum_{\alpha}\left|\left(n_{\alpha}\right)_{x}\right|^{4}=(2 / 3) n_{H}^{4}$

- $\sum_{l \neq m}\left|\left(n_{l}\right)_{x}\right|^{2}\left|\left(n_{m}\right)_{x}\right|^{2}=4 n_{H}^{4}$

The $W$-energy is $\int n_{x}^{4}=(14 / 3) n_{H}^{4}, \int n_{z}^{4}=n_{0}^{4}+8 n_{0}^{2} n_{H}^{2}+$ $(14 / 3) n_{H}^{4}$ and

$$
\mathrm{FCC}: W\left(n_{0}^{4}+8 n_{0}^{2} n_{H}^{2}+14 n_{H}^{4}\right)
$$

BCC state has

$$
\begin{aligned}
& 4 n_{0} \int \Phi_{z}^{3}=\sum_{\mathbf{q}_{1}+\mathbf{q}_{2}+\mathbf{q}_{3}=\mathbf{0}} n_{0}\left(n_{\mathbf{q}_{1}}\right)_{z}\left(n_{\mathbf{q}_{2}}\right)_{z}\left(n_{\mathbf{q}_{3}}\right)_{z} \\
& =3 n_{0} n_{H}^{3}\left[\cos \left(\theta_{1}+\theta_{4}-\theta_{5}\right)+\cos \left(\theta_{3}+\theta_{6}-\theta_{1}\right)\right. \\
& \left.\quad-\cos \left(\theta_{2}+\theta_{5}-\theta_{3}\right)-\cos \left(\theta_{2}+\theta_{4}+\theta_{6}\right)\right] .
\end{aligned}
$$

Calculation of $\int \Phi_{x}^{4}=\int \Phi_{y}^{4}=\int \Phi_{z}^{4}=(87 / 8) n_{H}^{4}$ can be obtained from

- $\sum_{\alpha}\left|\left(n_{\alpha}\right)_{x}\right|^{4}=(9 / 8) n_{H}^{4}$

- $\sum_{l \neq m}\left|\left(n_{l}\right)_{x}\right|^{2}\left|\left(n_{m}\right)_{x}\right|^{2}=(78 / 8) n_{H}^{4}$

The $W$-energy is $\int n_{x}^{4}=(87 / 8) n_{H}^{4}$ and

$$
\begin{aligned}
\mathrm{BCC}: & W\left(n_{0}^{4}+12 n_{0}^{2} n_{H}^{2}+\frac{261}{8} n_{H}^{4}\right. \\
+ & 3 n_{0} n_{H}^{3}\left[\cos \left(\theta_{1}+\theta_{4}-\theta_{5}\right)+\cos \left(\theta_{3}+\theta_{6}-\theta_{1}\right)\right. \\
& \left.\left.-\cos \left(\theta_{2}-\theta_{5}-\theta_{3}\right)-\cos \left(\theta_{2}+\theta_{4}+\theta_{6}\right)\right]\right)
\end{aligned}
$$

\section{Appendix D: Minimizing the phase angles}

The three phase angles of SkX3 enter in the energy as

$$
(9 U+6 W) n_{0} n_{H}^{3} \cos \left(\theta_{1}+\theta_{2}+\theta_{3}\right)
$$

It is minimized by choosing $\theta_{1}+\theta_{2}+\theta_{3}=\pi$.

The six phase angles appearing in the BCC energy can be grouped as

$$
\begin{aligned}
& X\left[\cos \left(\theta_{1}+\theta_{4}-\theta_{5}+\alpha_{1}\right)+\cos \left(\theta_{3}+\theta_{6}-\theta_{1}+\alpha_{2}\right)\right. \\
& \left.+\cos \left(\theta_{2}+\theta_{5}-\theta_{3}+\alpha_{3}\right)+\cos \left(\theta_{2}+\theta_{4}+\theta_{6}+\alpha_{4}\right)\right]
\end{aligned}
$$

with $X=\sqrt{27 U^{2}+30 U W+9 W^{2}}$ and $\alpha_{i}$ that can be worked out. The minimum energy configuration is achieved by taking all the arguments of the cosines equal to $\pi$. The energy of BCC coming from the $U$ and $W$ terms after minimizing the phase angles are

$$
\begin{aligned}
\mathrm{BCC}: & U\left(n_{0}^{4}+14 n_{0}^{2} n_{H}^{2}+54 n_{H}^{4}\right) \\
& +W\left(n_{0}^{4}+12 n_{0}^{2} n_{H}^{2}+\frac{261}{8} n_{H}^{4}\right) \\
& -4 \sqrt{27 U^{2}+30 U W+9 W^{2}} n_{0} n_{H}^{3}
\end{aligned}
$$

No other candidate states besides SkX3 and BCC have the phase angles entering in the energy. 
1 T. H. R. Skyrme, Proc. Roy. Soc. (London) A 260, 127 (1961); Nuc. Phys. 31, 556 (1962).

2 R. Rajaraman, Solitons and Instasntons, Chap. 3 (North Holland, 1987).

3 A. M. Polyakov, Phys. Lett. 59B, 79 (1975).

4 N. Nagaosa, Quantum Field Theory in Strongly Correlated Electronic Systems, Chap. 2 (Springer, 1999).

5 J. M. Kosterlitz and D. J. Thouless, J. Phys. C 6, 1181 (1972).

6 S. Mühlbauer, B. Binz, F. Joinetz, C. Pfleiderer, A. Rosch, A. Neubauer, R. Georgii, and P. Böni, Science 323, 915 (2009).

7 W. Munzer, A. Neubauer, T. Adams, S. Muhlbauer, C. Franz, F. Jonietz, R. Georgii, P. Boni, B. Pedersen, M. Schmidt, A. Rosch, and C. Pfleiderer, Phys. Rev. B 81, 041203(R) (2010).

${ }^{8}$ C. Pfleiderer, T. Adams, A. Bauer, W. Biberacher, B. Binz, F. Birkelbach, P. Böni, C. Franz, R. Georgii, M. Janoschek, F. Jonietz, T. Keller, R. Ritz, S. Mühlbauer, W. Münzer, A. Neubauer, B. Pedersen, and A. Rosch, J. Phys.:Condens. Matter 22, 164207 (2010).

9 X. Z. Yu, Y. Onose, N. Kanazawa, J. H. Park, J. H. Han, Y. Matsui, N. Nagaosa, and Y. Tokura, Nature (London) 465, 901 (2010).

10 Minhyea Lee, W. Kang, Y. Onose, Y. Tokura, and N. P. Ong, Phys. Rev. Lett. 102, 186601 (2009).

11 A. Neubauer, C. Pfleiderer, B. Binz, A. Rosch, R. Ritz, P. G. Niklowitz, and P. Böni, Phys. Rev. Lett. 102, 186602 (2009).

12 A. N. Bogdanov and D. A. Yablonskii, Sov. Phys. JETP
68, 101 (1989)

13 A. Bogdanov and A. Hubert, J. Magn. Magn. Mater. 138, 255 (1994).

$14 \mathrm{Su}$ Do Yi, Shigeki Onoda, Naoto Nagaosa, and Jung Hoon Han, Phys. Rev. B 80, 054416 (2009).

15 Jung Hoon Han, Jiadong Zang, Zhihua Yang, Jin-Hong Park, and Naoto Nagaosa, arXiv:1006.3973v2 (2010).

16 U. K. Roßler, A. N. Bogdanov, and C. Pfleiderer, Nature 442, 797 (2006).

17 A. A. Leonov, A. N. Bogdanov, U. K. Rößler, arXiv:1001.1292 (2010).

18 B. Binz, A. Vishwanath, and V. Aji, Phys. Rev. Lett. 96, 207202 (2006); B. Binz and A. Vishwanath, Phys. Rev. B 74, 214408 (2006).

19 Per Bak and M. Høgh Jensen, J. Phys. C 13, L881 (1980).

20 C. Pfleiderer, D. Reznik, L. Pintschovius, H. v. Löhneysen, M. Garst, and A. Rosch, Nature 427, 227 (2004).

21 Jinwu Ye, Y. B. Kim, A. J. Millis, B. I. Shraiman, P. Majumdar, and Z. Tesanović, Phys. Rev. Lett. 83, 3737 (1999); S. H. Chun et al., Phys. Rev. Lett. 84, 757 (2000); Masaru Onoda, Gen Tatara, and Naoto Nagaosa, J. Phys. Soc. Jpn. 73, 2624 (2004); P. Bruno, V. K. Dugaev, and M. Taillefumier, Phys. Rev. Lett. 93, 096806 (2004).

22 B. Binz and A. Vishwanath, Physica B 403, 1336 (2008).

23 T. H. R. Skyrme, Proc. Roy. Soc. London A 260, 127 (1961); Nuc. Phys. 31, 556 (1962).

24 I. Klebanov, Nuc. Phys. B262, 133 (1985); M. Kugler and S. Shtrikman, Phys. Lett. B 208, 491 (1988). 